\title{
Direct Writing of Photonic Structures by Two-Photon Polymerization
}

\author{
Yan Li*, ZhaoPei Liu, HaiBo Cui, Yun-Feng Xiao, Hong Yang and Qihuang Gong \\ Physics Department \& State Key Laboratory for Mesoscopic Physics, Peking University, \\ Beijing, 100871, P.R.China \\ Email address: li@pku.edu.cn
}

\begin{abstract}
Single-mode dielectric-loaded surface plasmon-polariton nanowaveguides with strong mode confinement at excitation wavelength of $830 \mathrm{~nm}$ and high- $Q$ polymer whispering gallery mode microcavities with surface roughness less than $12 \mathrm{~nm}$ have been directly written by two-photon polymerization, which pave the way to fabricate 3D plasmonic photonic structures by direct laser writing.
\end{abstract}

\section{Introduction}

Confinement and manipulation of photons using microcavities like whispering gallery mode (WGM) microcavities have triggered intense research interest. Recently, much attention has been paid to coupled photonic-plasmonic systems with a combination of photonic microcavities and metallic nanostructures that show a strong coupling between electromagnetic light modes of a resonator and collective electron oscillations (plasmons) in the metal. For example, we have proposed a kind of plasmonic WGM highly localized on the exterior surface of a metal-coated microresonator. The exterior mode can couple to an interior mode and this coupling produces a strong anticrossing behavior, which not only allows conversion of interior to exterior modes, but also forms a long-lived antisymmetric mode [1-3].

Due to the low cost and good biocompatibility of polymer materials, polymer WGM microcavities have attracted increasing interest. The fabrication of these microcavities is primarily based on the conventional lithography that is remarkably successful in producing planar devices via multiple steps. With the rapid development of femtosecond laser systems, direct laser writing by two-photon polymerization (TPP) has emerged as a powerful technique for creating fully 3D micro/nanostructures with sub-diffraction-limit feature-sizes, such as photonic crystals, metamaterials, waveguide-based devices, and MEMS [4-6].

Here we report the fabrication of dielectric-loaded surface plasmon-polariton (SPP) waveguides (DLSPPWs) on the gold film and high-Q polymer WGM microcavities by TPP. These DLSPPWs with optimal transverse dimensions around $250 \mathrm{~nm}$ are single-mode with strong mode confinement at excitation wavelength of $830 \mathrm{~nm}$. The quality factors of the fabricated WGM microcavities with surface roughness less than $12 \mathrm{~nm}$ are up to $1.48 \times 10^{5}$ limited by the material absorption.

\section{Experimental Results and Discussion}

The DLSPPWs were designed by the effective-index method (EIM) and then fabricated by TPP. The SPP guiding of the DLSPPWs was characterized by the leakage radiation microscopy (LRM). Figure 1 (a) shows a straight DLSPPW with a semicircular SPP launching structure. The images of sample and Fourier planes were captured to calculate the propagation length and the mode effective index as shown in Fig. 1(b) and (c). The intensity distribution across the waveguide confirmed that this DLSPPW mode indeed was single-mode as expected by EIM calculation. By exponentially fitting the intensity along the core of 
waveguide in Fig.1 (d), the propagation length can be obtained as $8.2 \pm 0.5 \mu \mathrm{m}$. The effective index of DLSPPW mode was $\sim 1.22 \pm 0.03$. Both the results of the propagation length and the mode effective index were in good agreement with the theoretical values.

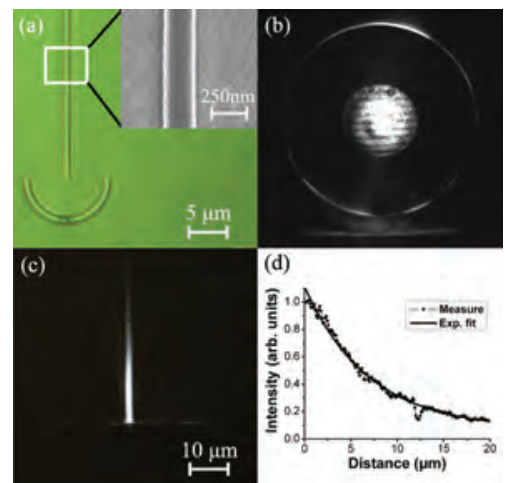

Fig. 1 (a) A straight DLSPPW with a semicircular SPP launching structure. (b) The LRM image at Fourier plane. (c) The image of object plane when only DLSPPW mode was retained. (d) Exponential fit of the intensity along the core of waveguide in (c).

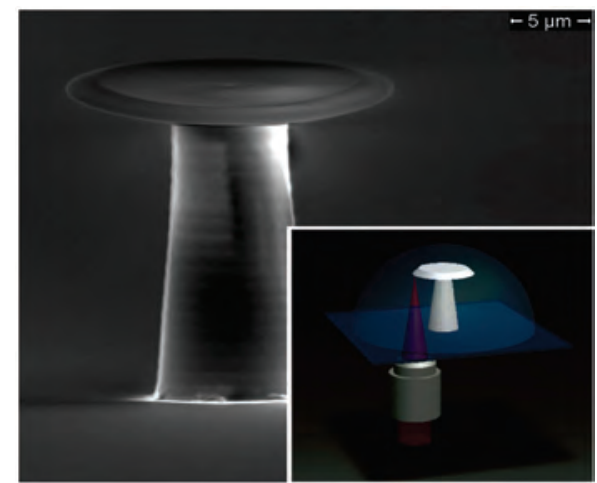

Fig. 2 SEM image of a fabricated microdisk cavity with surface roughness less than $12 \mathrm{~nm}$. Inset shows the schematic of the 3D micro-nano fabrication processing by two-photon polymerization.

The designed structure of a WGM microcavity is a polymer disk with a wedge edge to suppress the scattering loss. Figure 2 depicts a scanning electron microscopic (SEM) image of a microdisk. The inset shows the schematic of the 3D micro-nano fabrication processing by two-photon polymerization. The surface roughness was better than $12 \mathrm{~nm}$. Benefitting from the accurate position control of the laser focal spot in TPP, microdisk cavities with different sizes can be polymerized easily to investigate the parameter influence. The $Q$ factors can reach $1.48 \times 10^{5}$ in the $1550 \mathrm{~nm}$ band. It demonstrates that polymer absorption limited quality factors can be obtained in microdisk cavities fabricated with TPP. The integration of WGM microcavities and plasmons is under investigation.

\section{Conclusion}

We successfully fabricated dielectric-loaded surface plasmon-polariton waveguides and microdisk cavities using TPP technique. This technique opens new possibilities for fabrication of plasmonic microcavities with specific defects or a complex 3D coupled-cavity system for quantum simulation.

\section{REFERENCES:}

[1] Y.-F Xiao, C.L. Zou, et al, “High-Q exterior whispering-gallery modes in a metal-coated microresonator,” Phys. Rev. Lett. 105, 153902 (2010)

[2] X.F. Jiang, Y.-F. Xiao, et al, "Highly unidirectional emission and ultralow-threshold lasing from on-chip ultrahigh-Q microcavities,” Adv. Mat. 24, OP260-OP264 (2012)

[3] Y.-F. Xiao, Y.C. Liu, et al, “Strongly enhanced light-matter interaction in a hybrid photonic-plasmonic resonator,” Phys. Rev. A 85, 031805 (2012)

[4] H. Luo, Y. Li, et al, “Dielectric-loaded surface plasmon-polariton nanowaveguides fabricated by two-photon polymerization,” Appl. Phys. A, 97: 709-712 (2009)

[5] Z.P. Liu, Y. Li et al, “Direct laser writing of whispering gallery microcavities by two-photon polymerization,” Appl. Phys. Lett. 97, 211105 (2010).

[6] H.B. Cui, Y. Li et al,“Controlling aspect ratios of suspended nanorods fabricated by multi-photon polymerization,” Appl. Phys. A 105, 897 (2011). 